\title{
The prevalence of respiratory pathogens in adults with community-acquired pneumonia in an outpatient cohort
}

\author{
Jing Chen \\ Xiaoguang Li \\ Wei Wang \\ Ying Jia \\ Fei Lin \\ Jie $\mathrm{Xu}$
}

Department of Infectious Disease, Peking University Third Hospital, Beijing,

People's Republic of China

\author{
This article was published in the following Dove Press journal: \\ Infection and Drug Resistance
}

\begin{abstract}
Purpose: Community-acquired pneumonia is a common illness worldwide. In adults, community-acquired bacterial pneumonia has been well studied, but viral pneumonia is less well understood. We designed this study to identify respiratory pathogens, including common pneumonia-causing bacteria, viruses and atypical pneumonia pathogens, using reverse transcription-polymerase chain reaction.
\end{abstract}

Patients and methods: We conducted a retrospective study of outpatients with community-acquired pneumonia at the Fever Clinic of Peking University Third Hospital. We collected sputum or throat swabs from patients diagnosed with community-acquired pneumonia. Multiplex real-time reverse transcription-polymerase chain reaction was performed for 20 pathogens, including 9 viruses, 3 atypical pathogens and 8 bacteria.

Results: There were 232 outpatients enrolled in our study, and 153 patients $(65.9 \%)$ had positive test results, of which $26.7 \%$ were viruses, $19.4 \%$ were atypical pathogens and $19.8 \%$ were bacteria. Mycoplasma pneumoniae infection was detected at the highest frequency (19.0\%), exceeding Streptococcus pneumoniae infection. The most commonly identified viral pathogens were IFVs (15.1\%), PIVs (3.4\%) and RhV (2.6\%). The most commonly identified bacteria were Streptococcus pneumoniae (9.1\%), Haemophilus influenza $(6.5 \%)$ and Klebsiella pneumoniae (2.6\%).

Conclusion: Our study suggests that viruses were commonly detected in outpatients with CAP, and IFVs were the most common viruses, especially during flu season. Patients with viral infection were prone to viral-bacterial coinfection. Mycoplasma pneumoniae was the leading pathogen in the outpatients with CAP. Viral infection occurs in a large number of outpatients with CAP, and it should receive greater attention in clinical work.

Keywords: community-acquired pneumonia, respiratory pathogens, Mycoplasma pneumoniae; viral pneumonia, outpatient

\section{Introduction}

Community-acquired pneumonia (CAP) is a common illness that affects millions of people each year, and it is one of the most common infectious diseases that can lead to morbidity and mortality worldwide. The Global Burden of Disease Study reported that lower respiratory tract infection remains the second largest cause of death and years of life lost in 2013. ${ }^{1}$ CAP is a disease that is diagnosed by clinical, epidemiological, radiographic and laboratory characteristics, and the clinician usually relies on empirical evidence when determining therapy, which can lead to antibiotic abuse. Therefore, the detection of respiratory pathogens is very important for the clinician to
Correspondence: Jie Xu

Department of Infectious Disease, Peking University Third Hospital, Beijing, People's Republic of China

Email bysyin@I63.com 
be able to treat CAP. Pathogens called bacteria, viruses, and fungi can cause pneumonia. In adults, bacterial pneumonia has been extensively studied, but viral pneumonia is not as well understood. With the help of new diagnostic technologies, viral respiratory tract infections are being identified as common etiologies of CAP. In adults, approximately 200 million cases of viral community-acquired pneumonia occur every year, and 15-56\% of CAP cases are associated with RVIs. ${ }^{2,3}$ We designed this study to identify pathogens, including common pneumonia-causing bacteria, viruses and atypical pathogens, by multiplex real-time reverse transcription-polymerase chain reaction (RT-PCR).

\section{Materials and methods Study patients}

The respiratory etiological surveillance monitoring system of the Centers for Disease Control (CDC) of Beijing is designed to monitor respiratory pathogens in Beijing.

Peking University Third hospital is a sentinel hospital responsible for routine surveillance of respiratory tract infections. We conducted a retrospective study of outpatients through routine surveillance with CAP from June 2017 to February 2019 in a fever clinic. The patients were enrolled according to the following criteria. CAP was diagnosed according to the diagnostic criteria of Chinese adult community-acquired pneumonia (2016 edition), developed by the Chinese Society of Respiratory Medicine: (1) fever (a body temperature $>38.0{ }^{\circ} \mathrm{C}$ ) or hypothermia (a body temperature $<35.5^{\circ} \mathrm{C}$ ); (2) leukocytosis (a white blood cell count $>10,000 / \mathrm{ml}$ ) or leukopenia (a white blood cell count $<4000 / \mathrm{ml}$ ); (3) signs/symptoms of cough, sputum, respiratory symptom aggravation (with or without purulent sputum), chest pain, dyspnea and hemoptysis; (4) chest radiological imaging features such as patchy infiltration, leaf segment consolidation shadow, and interstitial inflammation change (with or without pleural effusion); and (5) signs of lung consolidation or rales on lung auscultation. CAP can be established when excluding tuberculosis, pulmonary tumor, noninfectious pulmonary interstitial disease, pulmonary edema, pulmonary atelectasis, pulmonary embolism, pulmonary eosinophil infiltration and pulmonary vasculitis.

\section{Data and specimen collection}

Detailed demographic information was documented, and laboratory data were collected from the patients' medical files. We collected sputum, or throat swabs if there was no sputum, from patients diagnosed with CAP. Specimens were transported to the CDC laboratory for the pathogen nucleic acid amplification test by RT-PCR for 20 pathogens, including 9 viruses, 3 atypical pathogens and 8 bacteria. The pathogens were human rhinovirus (HRV), influenza A virus (IFA), influenza B virus (IFB), human coronavirus (HCoV) (229E/HKU1 and OC43/NL63), adenoviruses (ADVs), respiratory syncytial virus (RSV), parainfluenza viruses (PIVs), human metapneumovirus (hMPV), enteroviruses (EVs), Mycoplasma pneumoniae, Chlamydia pneumoniae, Legionella spp., Pyogenic streptococcus, Staphylococcus aureus, Klebsiella pneumoniae, Escherichia coli, Streptococcus pneumoniae, Pseudomonas aeruginosa, Moraxella catarrhalis and Haemophilus influenzae.

\section{Statistical analysis}

All analyses were performed with Statistical 19.0 and Microsoft Excel 2007. General data are presented as a percentage $(\mathrm{P})$ or mean $\pm \mathrm{SD}$. Differences in categorical variables between groups were compared using the $\chi 2$ test. A single-tailed $P$-value of $<0.05$ was considered statistically significant.

\section{Methods}

1. Nucleic Acid Extraction: Total nucleic acids, including DNA and RNA, were extracted from $200 \mu \mathrm{L}$ of each specimen using a magnetic bead nucleic acid extraction kit ((Thermo Scientific ${ }^{\mathrm{TM}}$ KingFisher ${ }^{\mathrm{TM}}$ Flex Magnetic Particle Processors, Thermo Fisher).

2. RT-PCR Screening for Respiratory Viruses: For all collected specimens, multiplex RT-PCR assays were performed using a commercially available RT-PCR Taq kit (Multiplex Combined Real-time PCR Detection Kit for Respiratory Viruses, Multiplex Combined Real-time PCR Detection Kit for Respiratory Bacteria, Jiangsu Uninovo Biological Technology Co. Ltd., China). The housekeeping gene RNaseP was used as the internal standard in the multiplex real-time RT-PCR assay. There were positive and negative controls in the RT-PCR Taq kit. In the detection kit for the respiratory viruses, positive controls were the artificially synthesized virus RNA with target sequences, and negative controls were RNasefree and DNase-free water. In the detection kit for the respiratory bacteria, positive controls were the bacterial pathogens and RNaseP of target sequence plasmids, and negative controls were RNase-free and DNase-free water. 
3. Results determination: The following conditions should be simultaneously met: 1 . sleek S-Curve; 2 . $\mathrm{CT} \leq 353 ; 3 . \Delta \mathrm{Rn}>1 \times 10^{4}$.

4. Limits of detection for the different pathogens: In the detection kit for the respiratory viruses, when the viral nucleic acid $<10^{3}$ in the reaction system, it may not be detected. Although the target sequences are in the highly conserved domain, the viral genes are always mutating, which can lead to false negatives.In the detection kit for the respiratory bacteria, when the bacterial nucleic acid $<10^{2}$ in the reaction system, it may not be detected. The target sequences are in the highly conserved domain; if the bacterial nucleic acid mutates in the target position, it may lead to false negatives.

\section{Results}

1. Characteristic of patients with CAP and prevalence of respiratory pathogens

There were 232 patients enrolled in our study, and 123 $(53.0 \%)$ were male and $109(47.0 \%)$ were female. The age of the patients was $42.9 \pm 18.5$ years, ranging from 18 to 85 years. Of the 232 samples, there were $109(47.0 \%)$ sputum specimens and $123(53.0 \%)$ throat specimens. An etiological diagnosis could be established in $153(65.9 \%$, 153/232) patients.

A viral diagnosis was made in $62(26.7 \%, 62 / 232)$ patients. The rate of single virus infection was $15.1 \%$ (35/232) and the polymicrobial infection (viruses + bacteria, viruses + atypical pathogens, and two viruses) was $11.6 \%$ (27/232).

Mycoplasma pneumoniae was detected in 44 samples $(19.0 \%, 44 / 232)$, and Chlamydia pneumoniae was only detected in 1 sample, while the rate of single Mycoplasma pneumoniae infection was $12.5 \%$ (29/232). A bacterial diagnosis was made in $46(19.8 \%, 46 / 232)$ patients, while the rate of single bacterial infection was $7.8 \%(18 / 232)$, and the polymicrobial infection (bacteria + viruses, bacteria + atypical pathogens) was $12.1 \%$ (28/ 232). The number of bacteria plus atypical pathogens was 17 , and the number of bacteria plus viruses was 11 .

The most commonly identified viral pathogens were IFVs [33 (IFA=26, 11.2\% and IFB=9, 3.9\%), 15.1\%], PIVs $(8,3.4 \%)$, and $\mathrm{RhV}(6,2.6 \%)$. The most commonly identified nonviral pathogens were Mycoplasma pneumoniae $(44,19.0 \%)$, Streptococcus pneumoniae (21, 9.1\%), Haemophilus influenza $(15,6.5 \%)$ and Klebsiella pneumoniae $(6,2.6 \%)$ (Table 1).

\section{The age distribution of respiratory pathogens}

To determine whether the etiological agent for CAP varied with age, we compared the prevalence of respiratory pathogens in different age groups. We divided the patients into two groups: patients $<65$ and $\geq 65$ years old. In the group of patients $<65$ years old, the rates of viruses, atypical pathogens and bacteria were $23.6 \%$ (45/191), 23.0\% (44/191) and $20.4 \%(39 / 191)$, respectively. In the group of patients $\geq 65$ years old, the rates of viruses, atypical pathogens and bacteria were $41.5 \%$ (17/41), 2.4\% (1/41) and 14.6\% (6/41), respectively. In the group of patients $\geq 65$ years old, viral infection was the majority, while in the group of patients $<65$ years old, viruses, atypical pathogens and bacteria were all the main pathogens in CAP.

In the nucleic acid test for 20 pathogens, the rate of MP was higher in the patients $\geq 65$ years old, and the rate of hMPV in the patients $\geq 65$ was higher $(P<0.05)$. There was no significant difference between the two groups regarding the other 18 pathogens (Table 1, Figure 1).

\section{The polymicrobial infection of pathogens}

There were a total of 41 polymicrobial infections of the pathogens. The mixed viral infection (viral + bacterial or viral + atypical pathogens) comprised vast majority $(61.0 \%, 25 / 41)$. Streptococcus pneumoniae was the most common bacteria cooccurring with virus, followed by Haemophilus influenza and Mycoplasma. In the mixed infection of Mycoplasma and bacterial pathogens $(26.8 \%, 11 / 41)$, Klebsiella pneumoniae was the most common bacteria co-occurring with Mycoplasma, followed by Streptococcus pneumoniae and Haemophilus influenzae. In the analysis of multiple bacterial pathogen infections (12.2\%, 5/41), Streptococcus pneumoniae plus Haemophilus influenzae was the most prevalent combination. There were only two samples with double viral pathogen infections. (Figure 2).

\section{Monthly and seasonal distribution of pathogens}

Mycoplasma pneumoniae was the most frequently detected infection in CAP and throughout the year. Infection with this pathogen was highest during August, September, October and November, which are late summer and autumn in China. Influenza virus was highest in January and December, which are winter months. PIVs were detected almost from April to October. In August, 
Table I The prevalence of the respiratory pathogens and the analysis grouped by age

\begin{tabular}{|c|c|c|c|c|}
\hline & Pathogens & Subjects $(n=253)$ & $<65$ years $(n=191)$ & $\geq 65$ years $(n=41)$ \\
\hline \multirow[t]{10}{*}{ Virus } & IFA & 26 (11.1\%) & $20(10.5 \%)$ & $6(14.6 \%)$ \\
\hline & IFB & $9(3.6 \%)$ & $6(3.1 \%)$ & $3(7.3 \%)$ \\
\hline & PIVs & $8(3.2 \%)$ & $6(3.1 \%)$ & $2(4.9 \%)$ \\
\hline & RhV & $6(2.4 \%)$ & $4(2.1 \%)$ & $2(4.9 \%)$ \\
\hline & EV & $4(1.6 \%)$ & $4(2.1 \%)$ & 0 \\
\hline & hMPV* & $4(1.6 \%)$ & I $(0.5 \%)$ & $3(7.3 \%)$ \\
\hline & RSV & $3(1.2 \%)$ & $3(1.6 \%)$ & 0 \\
\hline & ADV & I (0.4\%) & I (0.5\%) & 0 \\
\hline & $\mathrm{HCoV}$ & I (0.4\%) & 0 & I (2.4\%) \\
\hline & All & $62(24.5 \%)$ & $45(23.6 \%)$ & $17(41.5 \%)$ \\
\hline \multirow[t]{4}{*}{ Atypical pathogens } & $\begin{array}{l}\text { Mycoplasm } \\
\text { pneumoniae* }\end{array}$ & 44 (I7.4\%) & 43 (22.5\%) & I (2.4\%) \\
\hline & Chlamydia pneumoniae* & I $(0.4 \%)$ & I $(0.5 \%)$ & 0 \\
\hline & Legionella spp & 0 & 0 & 0 \\
\hline & All & 45 (I7.8\%) & $44(23.0 \%)$ & I \\
\hline \multirow[t]{9}{*}{ Bacteria } & - Streptococcus Pneumoniae & 21 (8.3\%) & 17 (8.9\%) & $4(9.8 \%)$ \\
\hline & Haemophilus Influenzae & $15(6.2 \%)$ & $13(6.8 \%)$ & $2(4.9 \%)$ \\
\hline & Klebsiella Pneumoniae & $6(2.4 \%)$ & $6(3.1 \%)$ & 0 \\
\hline & - Streptococcus Pyogenes & I (0.4\%) & I (0.5\%) & 0 \\
\hline & Moraxella Catarrhalis & I $(0.4 \%)$ & I $(0.5 \%)$ & 0 \\
\hline & Pseudomonas Aeruginosa & I $(0.4 \%)$ & I $(0.5 \%)$ & 0 \\
\hline & Staphylococcus Aureus & 0 & 0 & 0 \\
\hline & Escherichia Coli & 0 & 0 & 0 \\
\hline & All & 45 & 39 & 6 \\
\hline
\end{tabular}

Note: $* P<0.05$.

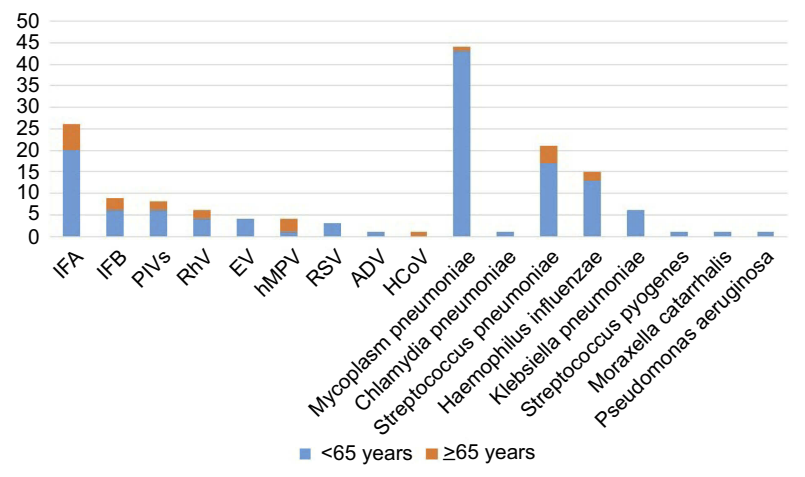

Figure I Age distribution of respiratory pathogens profiles.

we detected most types of viruses, including IFA, hMPV, PIVs, HRVs and EVs. We did not find Legionella in our study of CAP (Figures 3-5).

1. The influence of specimen type on positive specimens for different pathogens

There were sputum and throat swab specimens in our study, and we analyzed the influence of specimen type on
Table 2 The influence of specimen type in the positive specimens for the different pathogens

\begin{tabular}{|l|l|l|}
\hline \multirow{2}{*}{ pathogens } & \multicolumn{2}{|c|}{ Number of patients } \\
\cline { 2 - 3 } & $\begin{array}{l}\text { Sputum } \\
(\mathbf{n}=109)\end{array}$ & $\begin{array}{l}\text { Throat swab } \\
(\mathbf{n}=124)\end{array}$ \\
\hline IFA & $14(12.8 \%)$ & $12(9.7 \%)$ \\
IFB & $6(5.5 \%)$ & $3(2.4 \%)$ \\
PIVs & $6(5.5 \%)$ & $2(1.6 \%)$ \\
RhV* & $6(5.5 \%)$ & 0 \\
EV & $2(1.8 \%)$ & $2(1.6 \%)$ \\
hMPV & $3(2.8 \%)$ & $1(0.8 \%)$ \\
RSV & $2(1.8 \%)$ & $1(0.8 \%)$ \\
ADV & $1(0.9 \%)$ & 0 \\
HCoV & $1(0.9 \%)$ & 0 \\
Mycoplasm pneumonia** & $40(36.7 \%)$ & $4(3.2 \%)$ \\
Chlamydia pneumoniae & $1(0.9 \%)$ & 0 \\
Legionella spp & 0 & 0 \\
\hline
\end{tabular}

Note: $* P<0.05$.

the positive specimens for the different pathogens. The positive detection rate was higher in the sputum specimens, and there was a difference of statistics in RhV and 
A

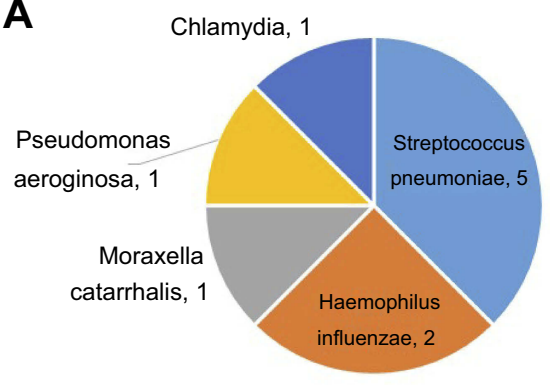

C

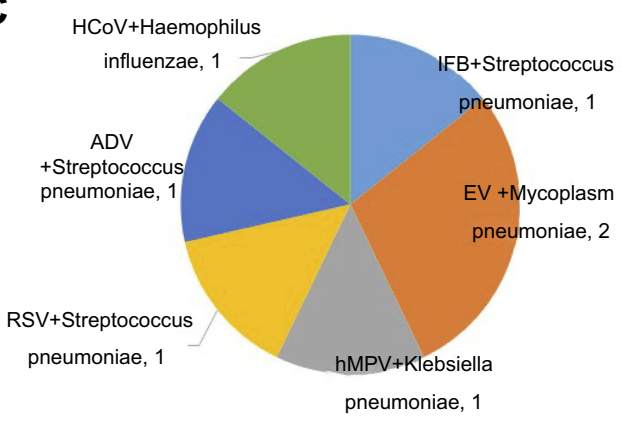

$\mathbf{E}$

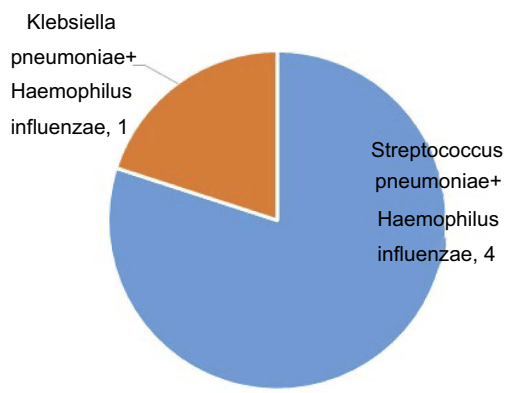

B

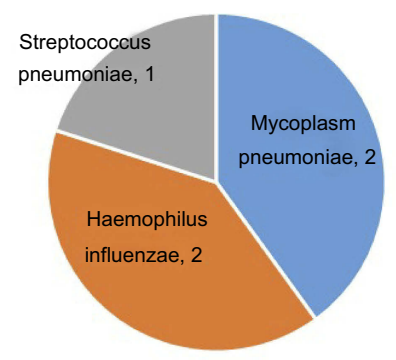

D Pseudomonas

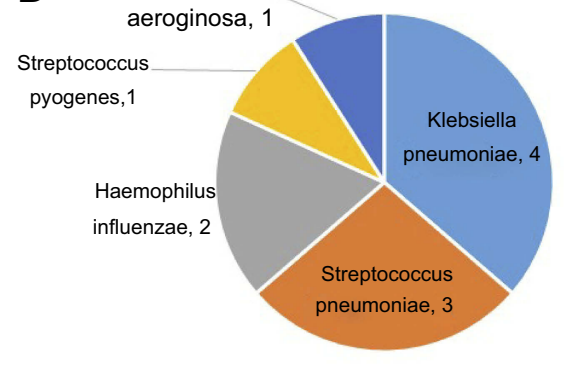

$\mathbf{F}$

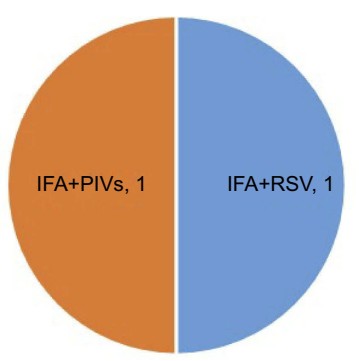

Figure 2 The polymicrobial infection of the pathogens by numbers of positive specimens. (A) mixed IFA and bacterial or atypical pathogens. (B) mixed RhV and bacterial or atypical pathogens. (C) other virus mixed with single bacterial or atypical pathogens. (D) mixed Mycoplasm pneumoniae and bacterial pathogens. (E) multiple bacterial pathogens. (F) multiple viral pathogen.

Mycoplasma pneumoniae $(P<0.05)$. The positive detection rate in the sputum and throat swabs were $5.5 \%$ vs 0 for $\mathrm{RhV}$ and $36.7 \%$ vs $3.2 \%$ for Mycoplasma pneumoniae, respectively (Table 2).

\section{Discussion}

We detected 20 common respiratory pathogens, including 9 viruses, 3 atypical pathogens and 8 bacteria, by RT-PCR in this study, and our findings showed that $65.9 \%$ had positive test results, of which $26.7 \%$ were viruses, $19.4 \%$ were atypical pneumonia and $19.8 \%$ were bacteria. Viral infection played an important role in CAP. The most commonly identified viral pathogens were IFVs (15.1\%),
PIVs (3.4\%) and RhV (2.6\%). The most commonly identified nonviral pathogens were Mycoplasma pneumoniae (19.0\%), Streptococcus pneumoniae (9.1\%), Haemophilus influenza (6.5\%) and Klebsiella pneumoniae (2.6\%). In a previous epidemiological survey in China, Mycoplasma pneumoniae and Streptococcus pneumoniae were the two main pathogens found in CAP, followed by Haemophilus influenza, Chlamydia pneumoniae, Klebsiella pneumoniae and Staphylococcus aureus. With the development of virus detection technology, clinicians can pay more attention to viral infections. The virus detection rate in CAP of Chinese adults is $15.0-34.9 \%$, and the most common viruses are influenza viruses, followed by PIVs, RhV, 


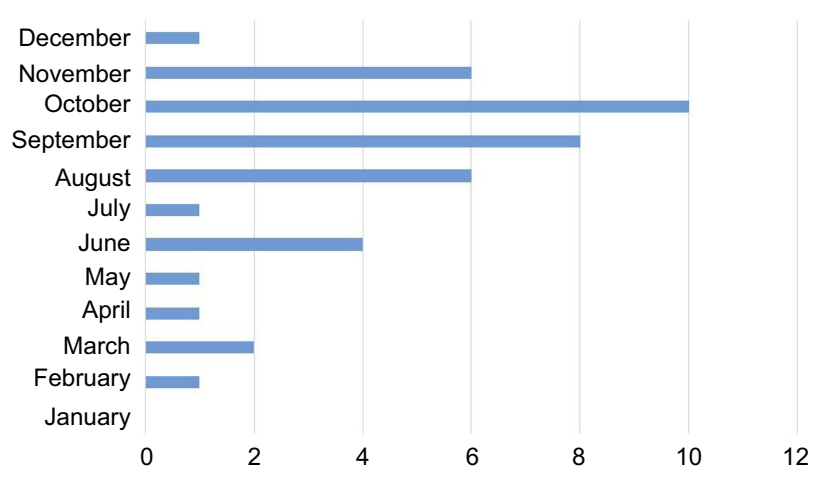

Figure 3 Monthly distribution of Mycoplasma pneumoniae by numbers of positive specimens.

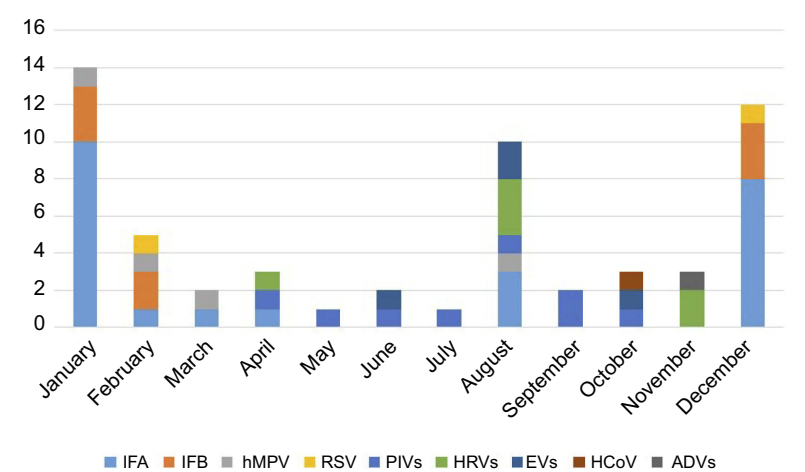

Figure 4 Monthly distribution of viral pathogens by numbers of positive specimens.

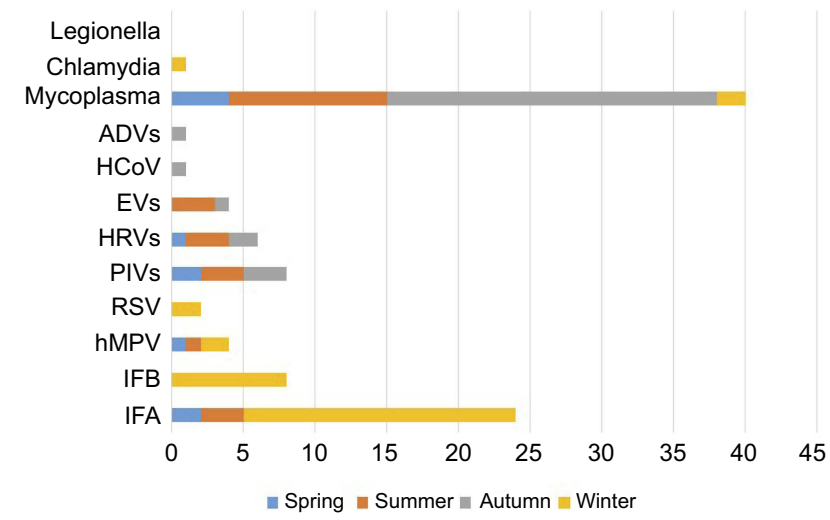

Figure 5 Seasonal distribution of viral and atypical pathogens by numbers of positive specimens.

ADV, hMPV and $\mathrm{RSV}^{4-7}$ In our study, the detection rate of viral infection was $26.7 \%$, and IFVs, PIVs, and RhV were the main viruses. This result was consistent with previous research findings in Chinese adults. In another CAP study of the Japanese population, the most frequently identified pathogens were Streptococcus pneumoniae (24.6\%), Haemophilus influenza (18.5\%), and Chlamydia pneumoniae (6.5\%), and Mycoplasma pneumoniae in
$5.2 \%$. The frequency of atypical pathogens reached $16.5 \%$, and the virus frequency reached $16.4 \% .^{5,8}$ The composition of respiratory pathogens differs from our country.

Of those patients who tested positive for viruses, 25 $(10.8 \%, 25 / 232)$ had polymicrobial infections, 6 had viruses plus atypical pathogens, and 19 had viruses plus bacteria. In some Chinese studies, the rate of mixed viral and bacterial or atypical pathogens was $5.8-65.7 \%{ }^{4}$ Our study demonstrated that nearly half of the patients with viral infection had polymicrobial infections, and the polymicrobial infection demand our attention in clinical work.

Mycoplasma pneumoniae infection had the highest frequency of detection in CAP and throughout the year $(18.9 \%)$ in the outpatient study. In a multicenter study of CAP in a Chinese population, Mycoplasmal pneumonia accounted for $20.7 \%$, and it was the most common pathogen, exceeding Streptococcus pneumoniae. ${ }^{9}$ In recent studies, Mycoplasma pneumoniae seems to comprise a larger proportion of respiratory pathogens. In our clinical work, we should consider Mycoplasma pneumoniae and make the selection of outpatient antibiotics more targeted.

There were some limitations in our study. First, the detection method of bacteria was a limitation because of the absence of urinary testing, serological testing and blood culture, which can detect many additional cases of bacterial pneumonia. Second, it was an outpatient cohort study, and we could only collect sputum and throat swab specimens. Bronchoalveolar lavage specimens, which may raise the detection rate of respiratory pathogens, could not be collected. We can improve the design method for better detection of respiratory pathogens in the future.

\section{Conclusion}

In conclusion, our study suggests that viruses were commonly detected in outpatients with CAP, and IFVs were the most common virus, especially during the flu season. Patients with viral infection were prone to viral-bacterial coinfection. Mycoplasma pneumoniae infection was the leading pathogen in the outpatients with CAP. Virals infection occurs in a large proportion of the outpatients with $\mathrm{CAP}$, and it should receive greater attention in clinical work.

\section{Ethical approval and consent to participate}

General ethics approval has been conducted by the Centers for Disease Control (CDC) in Beijing and the respiratory 
etiological surveillance monitoring system. This study was approved by the Ethics Committee of the Centers for Disease Control in Beijing. The patients enrolled signed the consent to participate. The funding program fro this study was the Major National Science and Technology Project of China (2017ZX 10103004)

\section{Disclosure}

The authors report no conflicts of interest in this work.

\section{References}

1. GBD 2013 Mortality and Causes of Death Collaborators. Global, regional, and national age-sex specific all-cause and cause-specific mortality for 240 causes of death, 1990-2013: a systematic analysis for the global burden of disease study 2013. Lancet. 2015;385:117171. doi:10.1016/S0140-6736(14)61682-2

2. Ruuskanen O, Lahti E, Jennings LC, Murdoch DR. Viral pneumonia. Lancet. 2011;377(9773):1264-1275. doi:10.1016/S0140-6736(10) 61459-6
3. Liu J, Ai HW, Xiong Y, et al. Prevalence and correlation of infectious agents in hospitalized children with acute respiratory tract infections in central China. PLoS One. 2015;10(3): e0119170. doi:10.1371/journal. pone. 0119170

4. Respiratory Society of Chinese Medical Association. The diagnostic and treatment guideline of Chinese adult with community acquired pneumonia (2006). Chin J Tuberc Respir Dis. 2006;39(4):253-279.

5. Cao B, Ren LL, Zhao F, et al. Viral and mycoplasma pneumoniae community-acquired pneumonia in fever outpatients. Exp Biol Med (Maywood). 2012;237(11):1256-1261.

6. Qu JX, Gu L, Pu ZH, et al. Viral etiology of community-acquired pneumonia among adolescents and adults with mild or moderate severity and its relation to age and severity. BMC Infect Dis. 2015;15:89. doi:10.1186/s12879-015-0808-0

7. Zhan Y, Yang Z, Chen R, et al. Respiratory virus is a real pathogen in immunocompetent community-acquired pneumonia: comparing to influenza like illness and volunteer controls. BMC Pulm Med. 2014;14(1): 144.

8. Saito A, Kohno S, Matsushima T, et al. Prospective multicenter study of the causative organisms of community-acquired pneumonia in adults in Japan. J Infect Chemother. 2006;12:63-69. doi:10.1007/ s10156-005-0425-8

9. Liu YN, Chen MJ, Zhao TM. A multicentre study on the pathogenic agents in 665 adult patients with community-acquired pneumonia in cities of China. Chin J Tuberc Respir Dis. 2006;29:3-9.
Infection and Drug Resistance

\section{Publish your work in this journal}

Infection and Drug Resistance is an international, peer-reviewed openaccess journal that focuses on the optimal treatment of infection (bacterial, fungal and viral) and the development and institution of preventive strategies to minimize the development and spread of resistance. The journal is specifically concerned with the epidemiology of antibiotic resistance and the mechanisms of resistance development and diffusion in both hospitals and the community. The manuscript management system is completely online and includes a very quick and fair peerreview system, which is all easy to use. Visit http://www.dovepress.com/ testimonials.php to read real quotes from published authors. 